CONFORMAL GEOMETRY AND DYNAMICS

An Electronic Journal of the American Mathematical Society

Volume 5, Pages 81-99 (September 6, 2001)

S $1088-4173(01) 00076-5$

\title{
BOUNDARY BEHAVIOR OF QUASI-REGULAR MAPS AND THE ISODIAMETRIC PROFILE
}

\author{
BRUCE HANSON, PEKKA KOSKELA, AND MARC TROYANOV
}

\begin{abstract}
We study obstructions for a quasi-regular mapping $f: M \rightarrow N$ of finite degree between Riemannian manifolds to blow up on or collapse on a non-trivial part of the boundary of $M$.
\end{abstract}

\section{INTRODUCTION}

1.1. Setting of the problem. A major result in quasi-conformal geometry states that a quasi-conformal map from a unit ball onto itself extends to the boundary sphere. One may, in fact, state the following more general theorem:

Let $f: M \rightarrow N$ be a quasi-conformal map between oriented $n$-dimensional manifolds. Assume that $M$ and $N$ are the interiors of smooth, compact Riemannian manifolds $\bar{M}$ and $\bar{N}$ with boundaries $\partial M$ and $\partial N$, respectively. Then $f$ extends to a homeomorphism $\bar{f}: \bar{M} \rightarrow \bar{N}$. Furthermore, the trace of $f$ defines a quasi-conformal map $\partial f: \partial M \rightarrow \partial N$ if $n>2$.

This result was proved by Ahlfors in dimension 2 and by Gehring in dimension $\geq 3$; it is a fundamental step in Mostow's proof of his rigidity theorem for hyperbolic manifolds (see [1], [6] and [10]).

The compactness of $\bar{N}$ is an essential hypothesis in the above theorem. In this paper, we aim at extending this result in the absence of any assumptions about the boundary of $N$. For that, we will use the following definition as a generalized concept of "boundary behavior" of a map:

Definition 1. We say that $f$ blows up on the set $E \subset \partial M$ if $\lim _{x \rightarrow E} d\left(f\left(x_{0}\right), f(x)\right)=$ $\infty$ for any $x_{0} \in M$.

Similarly, $f$ is said to collapse on $E \subset \partial M$ if $\lim _{x_{1}, x_{2} \rightarrow E} d\left(f\left(x_{1}\right), f\left(x_{2}\right)\right)=0$.

Observe that these conditions are purely topological; one may think of the size of the largest set where $f$ collapses as a measure of the non-injectivity of the "boundary map" and the largest set where $f$ blows up as the "preimage of infinity".

Using the method of Gehring's paper, it is not difficult to obtain the following generalization of his theorem.

Theorem. Let $f: M \rightarrow N$ be a quasi-conformal map where $M$ and $N$ are the interiors of smooth manifolds $\bar{M}, \bar{N}$ with boundaries $\partial M, \partial N$.

Then $f$ has a continuous extension $\bar{f}: \bar{M} \rightarrow \bar{N}$ if and only if $f$ does not blow up at any point of $\partial M$.

Received by the editors June 4, 2001.

2000 Mathematics Subject Classification. Primary 30C65.

The second author was supported in part by the Academy of Finland grants 39788 and 41933.

(C)2001 American Mathematical Society 
A natural problem is now to find conditions which will guarantee that a quasiconformal map does not blow up or collapse on a large part of the boundary. An example of such a result is given by the following theorem which is a direct consequence of Theorems 1 and 2 below.

Theorem. Let $f: M \rightarrow N$ be a quasi-conformal map between oriented $n$-dimensional Riemannian manifolds. Assume that $N$ is complete with non-negative Ricci curvature and that $M$ is the interior of a smooth manifold with boundary $\partial M$. Then $f$ neither collapses nor blows up on any subset $E \subset \partial M$ of positive $(n-1)$ dimensional measure.

In the present paper, we prove such results not only for quasi-conformal maps, but for the more general class of quasi-regular mappings of finite degree.

1.2. Statements of the central results. Throughout the paper, $M$ and $N$ will always be connected, oriented $n$-dimensional Riemannian manifolds. We will also assume that $M$ is the interior of a smooth Riemannian manifold $\bar{M}$ with boundary, $\bar{M}=M \cup \partial M$.

We also suppose that $f: M \rightarrow N$ is a quasi-regular mapping with finite degree 1 . Recall that a continuous mapping $f: M \rightarrow N$ is quasi-regular if $f \in W_{l o c}^{1, n}(M, N)$ and there exists a constant $K$ such that $\left|d f_{x}\right|^{n} \leq K J_{f}(x)$ a.e. where $J_{f}$ is the Jacobian of $f$.

To state our results, we will need the following geometric definition:

Definition 2. The isodiametric profile of a Riemannian manifold $(N, g)$ is the function $\eta:[0, \operatorname{diam}(N)) \rightarrow \overline{\mathbb{R}}_{+}=[0, \infty]$ defined by

$$
\eta(r):=\sup \{\operatorname{Vol}(A): A \Subset N \text { and } \operatorname{diam}(A) \leq r\} .
$$

Thus $\eta$ is the smallest function such that $\operatorname{diam}(A) \leq r \operatorname{implies} \operatorname{Vol}(A) \leq \eta(r)$. For instance, if $N=\mathbb{R}^{n}$, then $\eta(r)=\frac{\alpha_{n-1}}{n 2^{n}} r^{n}$ where $\alpha_{n-1}=\frac{2 \pi^{n / 2}}{\Gamma(n / 2)}$ is the area of the unit sphere $S^{n-1} \subset \mathbb{R}^{n}$ (see [4, page 69]).

The central results of the present paper are the following two theorems.

Theorem 1. Let $\bar{M}=M \cup \partial M$ be a connected Riemannian manifold with boundary and $f: M \rightarrow N$ be a non-constant quasi-regular map of finite degree where $N$ has infinite diameter. Let $\eta$ be the isodiametric profile of $N$ and assume that

$$
\int_{\alpha}^{\infty}\left(\frac{r}{\eta(r)}\right)^{1 /(n-1)} d r=\infty .
$$

Then $f$ cannot blow up on a subset $E \subset \partial M$ of positive $(n-1)$-Hausdorff measure.

Remark 1. If the manifold $N$ is complete, then the divergence of the integral (2) implies that the manifold $N$ is conformally parabolic (i.e., infinity has zero $n$ capacity) (see [15], 17]). In Section 4 we will see simple examples of quasi-conformal mappings onto a conformally hyperbolic manifold that blow up the entire boundary.

We do not know in general if there are any obstructions to the existence of a map $f: M \rightarrow N$ blowing up on the boundary when the target $N$ is conformally hyperbolic.

\footnotetext{
${ }^{1}$ There are several notions of degree or multiplicity of a mapping. For quasi-regular mappings these various notions coincide (see [9, Th. 3.10]).
} 
Theorem 2. Let $\bar{M}=M \cup \partial M$ be a connected Riemannian manifold with boundary and $f: M \rightarrow N$ be a non-constant quasi-regular map of finite degree. Let $\eta$ be the isodiametric profile of $N$ and assume that

$$
\int_{0}^{\epsilon}\left(\frac{r}{\eta(r)}\right)^{1 /(n-1)} d r=\infty
$$

Then $f$ cannot collapse on a subset $E \subset \partial M$ of positive $(n-1)$-Hausdorff measure.

Remark 2. The integral in (3) always diverges if there is a global lower bound for the Ricci curvature of $N$ (this follows from volume comparison, see e.g., [3] Th. $3.9]$ ). We thus have an obstruction to $f$ collapsing on the boundary under a mild geometric hypothesis on $N$.

From the proof of Theorem 1 we obtain the following corollary in the special case of the unit ball in Euclidean space.

Corollary 1. Let $f: B^{n} \rightarrow N$ be a non-constant quasi-regular map of finite degree. Assume that the isodiametric profile $\eta$ of $N$ satisfies (2). Then the image of the radius $[0, z)$ under $f$ has finite length for $(n-1)$-almost every $z \in \partial B^{n}$.

This corollary is essentially sharp already when $N$ is an Euclidean domain and $f$ is quasi-conformal. Indeed, in [8] a quasi-conformal mapping $f: B^{n} \rightarrow \mathbb{R}^{n}$ is constructed with the property that $f([0, z))$ has infinite length for each $z \in E$ where $E \subset \partial B^{n}$ is a subset of Hausdorff dimension $n-1$.

The divergence of the integrals $\int\left(\frac{r}{\eta(r)}\right)^{1 /(n-1)}$ in Theorems 1 and 2 turn out to be quite sharp assumptions. Notice first that when $\eta(r) \leq C r^{n}$, then the integrand is at least of the order $O\left(\frac{1}{r}\right)$ and the integrals thus diverge. In fact one can allow for $\eta(r) \leq C r^{n}\left(|\log r|^{n-1}+1\right)$.

Conversely, given any positive decreasing function $\chi:(0,1) \rightarrow \mathbb{R}_{+}$such that $\chi(r) r$ is increasing on $(0,1), \lim _{r \rightarrow 0} \chi(r)=\infty$, and

$$
\int_{0}^{1} \chi(r) d r<\infty
$$

we construct in Section 4 a continuous, positive function $\rho$ on $B^{n}$ so that

$$
\left(\frac{r}{\eta(r)}\right)^{1 /(n-1)} \geq \chi(r)
$$

and the identity mapping $I d:\left(B^{n}, g_{0}\right) \rightarrow\left(B^{n}, h_{\rho}\right)$ (where $\left.h_{\rho}=\rho(x) g_{0}\right)$ collapses on the entire boundary.

We also give a similar result regarding the blowing up phenomenon. In these constructions the function $\rho$ is radial and either decreases or increases exponentially fast.

This exponential behavior of $\rho$ is excluded when the conditions (4) and (5) below are satisfied.

In fact it is possible to improve Theorems 1 and 2 under the assumptions (4) and (5) (which is the setting of the paper [2]); we give such improvements in Section 5.

1.3. Comparison with previous recent results. As a simple example, let $M=$ $B^{n}$ be the standard unit ball in Euclidean space with its canonical metric $g_{0}=$ $\sum_{i} d x_{i}^{2}$ and let $N=B^{n}$ be the unit ball with the Riemannian metric $h_{\alpha}=\rho(x) g_{0}$ where $\rho=\rho_{\alpha}(x)=\left(1-|x|^{2}\right)^{\alpha}$. Then the identity map $I d:\left(B^{n}, g_{0}\right) \rightarrow\left(B^{n}, h_{\alpha}\right)$ 
collapses on the entire boundary $\partial B^{n}$ if $\alpha>0$ and blows up on the entire boundary if $\alpha<-1$.

This example may look artificial, however, in Section [6 we will show that, in dimension 2, such a mapping can be realized (up to a bi-Lipschitz transformation) as a quasi-conformal embedding of the disk $B^{2}$ into $\mathbb{R}^{3}$.

In the recent paper [2] the same map $I d:\left(B^{n}, g_{0}\right) \rightarrow\left(B^{n}, h_{\rho}\right)$, where $h_{\rho}=$ $\rho(x) g_{0}$, was studied under the assumption that $\rho: B^{n} \rightarrow \mathbb{R}_{+}$satisfies a Harnack inequality:

$$
A^{-1} \leq \frac{\rho\left(x_{1}\right)}{\rho\left(x_{2}\right)} \leq A
$$

whenever $x_{1}, x_{2}$ belong to some ball $B\left(x, \frac{1}{2}(1-|x|)\right) \subset B^{n}$ and a volume growth condition:

$$
\operatorname{Vol}\left(B_{\rho}(x, r)\right)=\int_{B_{\rho}(x, r)} \rho^{n} d m \leq A^{\prime} r^{n}
$$

for each ball $B_{\rho}(x, r)$ in the metric $\rho$. Here $A$ and $A^{\prime}$ are two constants.

The Harnack inequality is equivalent to assuming that the identity mapping $I d: M \rightarrow N$ is uniformly quasi-symmetric in each ball $B\left(x, \frac{1}{2}(1-|x|)\right)$.

Under this setting, the following result was proved in [2]:

Theorem. Under the assumptions (4) and (5) above, the identity mapping Id: $\left(B^{n}, g_{o}\right) \rightarrow\left(B^{n}, h\right)$ cannot blow up on any set $E \subset \partial M$ of positive Hausdorff dimension and cannot collapse on any set containing at least two points.

This example will be further developed in Sections 4 and 5 .

1.4. Organization of the paper. The paper is organized as follows. Section 2 contains preliminary facts about Riemannian manifolds and quasi-regular mappings together with a technical lemma. Theorems 1 and 2 are proved in Section 3 and in Section 4 we show the sharpness of both theorems. Section 5 gives an improvement on Theorems 1 and 2 in the case where the density $\rho$ has a polynomial decay or growth rate. Finally, in the last section of the paper, we show that (in the planar case) the simple example described in the beginning of 1.3 can be realized as a quasi-conformal mapping of the unit disk onto a (singular) manifold embedded in 3 -space.

\section{Preliminaries}

2.1. A theorem of Fuglede. Recall that the $p$-modulus of a family $\Gamma$ of curves in a Riemannian manifold $M$ is defined by

$$
\mathbb{M}_{p}(\Gamma)=\inf _{\varrho \in \mathcal{F}(\Gamma)} \int_{M} \varrho^{p}(x) d \mu(x),
$$

where $\mathcal{F}(\Gamma)$ is the set of all non-negative Borel-measurable functions $\varrho: M \rightarrow \mathbb{R}$ such that

$$
\int_{\gamma} \varrho d s \geq 1
$$

for every locally rectifiable curve $\gamma \in \Gamma$.

One says that a property defined on curves holds for $p$-modulus almost all curves if it holds for all curves except a family of $p$-modulus zero. 
Example. Let $\psi: U \times I \rightarrow M$ be a local diffeomorphism, where $U \subset \mathbb{R}^{n-1}$ and $I$ is an interval. Choose a Borel subset $E \subset U$ and define $\Gamma_{E}$ to be the set of curves $\gamma_{z}(s)=\psi(z, s) \in M$ where $z \in E$. Then one easily shows that

$$
\mathbb{M}_{p}\left(\Gamma_{E}\right)=0 \quad \text { iff } \quad \lambda^{n-1}(E)=0 .
$$

The next result is due to B. Fuglede [5] (see also $§ 28$ in [16]).

Theorem. Let $f: M \rightarrow N$ be a continuous mapping of class $W_{l o c}^{1, p}$. Then for p-modulus almost every absolutely continuous path $\gamma: I \rightarrow M$ we have

a) the path $f \circ \gamma: I \rightarrow N$ is absolutely continuous, and

b) Length $\left(\left.f \circ \gamma\right|_{[a, b]}\right)=\int_{a}^{b}\|d f(\gamma(t))\| d t$.

Remark. The papers [5] and [16] only state part (a) of the theorem, but they in fact also prove (b), as a careful reading of the text shows.

2.2. Geometry near the boundary. Many books on Riemannian geometry contain a description of the geometry near a submanifold (see e.g., section 3.6 in [3]). Such descriptions also hold near the boundary if the latter is a smooth submanifold. Let $(M, \partial M)$ be a smooth Riemannian manifold with boundary and let $E \subset \partial M$ be a subset of the boundary with $\bar{E}$ compact. For each $z \in E$ and small $s \geq 0$ we set

$$
\phi(z, s):=\exp _{z}\left(s \mathbf{n}_{z}\right)
$$

where $\mathbf{n} \in T(\partial M)^{\perp}$ is the inward pointing normal vector field of $\partial M$ and exp is the Riemannian exponential map. We know that if $\bar{E} \subset \partial M$ is compact, then there exists $\delta>0$ such that the map $\phi: E \times[0, \delta] \rightarrow M$ is injective. Furthermore, the point $p=\phi(z, s) \in M$ is at distance $s$ from $z$ and $t \rightarrow \phi(z, s t)$ is the unique minimal geodesic joining $p$ to $z$.

As an example, if $M=B^{n}$ is the Euclidean unit ball, then $\phi(z, s)=(1-s) z$ for all $z \in S^{n-1}=\partial B^{n}$ and $0 \leq s<1$.

Finally, we can find a constant $c$ such that the volume element $d \mu$ of $M$ satisfies

$$
\frac{1}{c} d \tau \otimes d s \leq\left. d \mu\right|_{\phi(E \times[0, \delta])} \leq c d \tau \otimes d s,
$$

where $d \tau$ denotes the $(n-1)$-dimensional Hausdorff measure on $E$ (i.e., $d \tau=$ $\left.\left.d \mathcal{H}^{n-1}\right|_{E}\right)$.

2.3. On quasi-regular mappings. Given a $K$-quasi-regular map $f: M \rightarrow N$, one defines a function $\rho: M \rightarrow \mathbb{R}$ by $\rho(x):=J_{f}(x)^{1 / n}$ if the Jacobian $J_{f}$ is defined at $x$ and $\rho(x)=0$ elsewhere. Then, by definition, we have almost everywhere

$$
\frac{1}{\sqrt[n]{K}}\left|d f_{x}\right| \leq \rho(x) \leq\left|d f_{x}\right| .
$$

We know furthermore from the theory of quasi-regular mappings that $\rho$ is Borel measurable and if $f$ is not constant, then $\rho>0$ almost everywhere.

By Fuglede's theorem, we have for $n$-modulus almost every path $\gamma:[a, b] \rightarrow M$

$$
K^{-1 / n} \operatorname{Length}(f \circ \gamma) \leq \int_{\gamma} \rho d s \leq \operatorname{Length}(f \circ \gamma)<\infty .
$$

In particular, given a compact set $E \subset \partial M$,

$$
\int_{s}^{\delta} \rho(\phi(z, t)) d t<\infty
$$


for all $0<s<\delta$ and $\mathcal{H}^{n-1}$-almost every $z \in E$.

We will also need the area formula

$$
\int_{A} J_{f}(x) d \mu(x)=\int_{N} \operatorname{card}\left(A \cap f^{-1}(y)\right) d \nu(y),
$$

which holds for any measurable subset $A \subset M$.

Suppose that $f$ has finite degree, i.e., there exists a number $\operatorname{deg}(f)<\infty$ such that

$$
\operatorname{card}\left(f^{-1}(y)\right) \leq \operatorname{deg}(f)
$$

for almost all $y \in N$. Then we have

$$
\int_{A} \rho^{n}(x) d \mu=\int_{A} J_{f}(x) d \mu \leq \operatorname{deg}(f) \int_{f A} d \nu=\operatorname{deg}(f) \operatorname{Vol}(f A) .
$$

For more information about quasi-regular mappings, we refer to [11] and [13].

2.4. A calculus lemma. One of the key ingredients in the proofs of Theorems 1 and 2 is the following result concerning positive, measurable functions. Although this result looks classical, we did not find it in the literature.

Lemma 1. Suppose that $\psi \in L_{\mathrm{loc}}^{1}(a, b)$ is a positive measurable function satisfying $0<r(t):=\int_{a}^{t} \psi(s) d s<\infty$ for every $t \in(a, b)$. Then for any $q \geq 2$ we have

$$
\int_{a}^{b}\left(\frac{\int_{a}^{t} \psi}{\int_{a}^{t} \psi^{q}}\right)^{\frac{1}{q-1}} \psi(t) d t=\int_{0}^{L}\left(\frac{r}{\int_{a}^{t} \psi^{q}}\right)^{\frac{1}{q-1}} d r \leq(b-a) 2^{\frac{q+1}{q-1}} .
$$

Here $L:=r(b)=\int_{a}^{b} \psi(s) d s$.

Proof. We consider the case $L=\infty$; the minor modifications needed to treat the case $L<\infty$ are left to the reader. Define

$$
H(r)=\left(\frac{\int_{a}^{t} \psi}{\int_{a}^{t} \psi^{q}}\right)^{\frac{1}{q-1}}
$$

we need to estimate the integral $\int_{0}^{\infty} H(r) d r$.

We first look at $\int_{0}^{1} H(r) d r$; for any $k \in \mathbb{N}$, choose $t_{k} \in(a, b)$ such that

$$
r\left(t_{k}\right)=\int_{a}^{t_{k}} \psi(s) d s=2^{-k},
$$

and set $a_{k}:=\left(t_{k}-t_{k+1}\right)$. Now suppose that $2^{-k} \leq r(t)<2^{-k+1}$, so that $t_{k} \leq t<$ $t_{k-1}$. Then

$$
H(r)=\left(\frac{\int_{a}^{t} \psi}{\int_{a}^{t} \psi^{q}}\right)^{\frac{1}{q-1}} \leq\left(\frac{\int_{a}^{t_{k-1}} \psi}{\int_{t_{k+1}}^{t_{k}} \psi^{q}}\right)^{\frac{1}{q-1}}=\left(\frac{2^{-k+1}}{\int_{t_{k+1}}^{t_{k}} \psi^{q}}\right)^{\frac{1}{q-1}} .
$$

On the other hand, we have from Hölder's inequality

$$
\int_{t_{k+1}}^{t_{k}} \psi^{q} \geq a_{k}^{1-q}\left(\int_{t_{k+1}}^{t_{k}} \psi\right)^{q}=a_{k}^{1-q}\left(2^{-k-1}\right)^{q},
$$

and hence

$$
H(r) \leq 2^{\frac{1-k}{q-1}}\left(a_{k}^{q-1} 2^{q(k+1)}\right)^{\frac{1}{q-1}}=a_{k} 2^{k} 2^{\frac{q+1}{q-1}}
$$


Therefore

$$
\int_{2^{-k}}^{2^{-k+1}} H(r) d r \leq 2^{\frac{q+1}{q-1}} a_{k}
$$

and we obtain the estimate

$$
\int_{0}^{1} H(r) d r=\sum_{k=1}^{\infty} \int_{2^{-k}}^{2^{-k+1}} H(r) d r \leq 2^{\frac{q+1}{q-1}} \sum_{k=1}^{\infty} a_{k} .
$$

We now estimate the integral $\int_{1}^{\infty} H(r) d r$ by a similar method: For any integer $k \geq-1$, we choose $y_{k} \in(a, b)$ such that

$$
\int_{a}^{y_{k}} \psi(s) d s=2^{k}
$$

and set $b_{k}:=\left(y_{k}-y_{k-1}\right)$. Suppose that $2^{k} \leq r(x)<2^{k+1}$ so that $y_{k} \leq x<y_{k+1}$. Then

$$
H(r)=\left(\frac{\int_{a}^{t} \psi}{\int_{a}^{t} \psi^{q}}\right)^{\frac{1}{q-1}} \leq\left(\frac{\int_{a}^{y_{k+1}} \psi}{\int_{y_{k-1}}^{y_{k}} \psi^{q}}\right)^{\frac{1}{q-1}} \leq\left(\frac{2^{k+1}}{\int_{y_{k-1}}^{y_{k}} \psi^{q}}\right)^{\frac{1}{q-1}} .
$$

By Hölder's inequality we have

$$
\int_{y_{k-1}}^{y_{k}} \psi^{q} \geq b_{k}^{1-q}\left(\int_{y_{k-1}}^{y_{k}} \psi\right)^{q} \geq b_{k}^{1-q}\left(2^{k-1}\right)^{q},
$$

so

$$
H(r) \leq 2^{-k} 2^{\frac{q+1}{q-1}} b_{k},
$$

and hence

$$
\int_{2^{k}}^{2^{k+1}} H(r) d r \leq 2^{\frac{q+1}{q-1}} b_{k}
$$

It follows that

$$
\int_{1}^{\infty} H(r) d r=\sum_{k=0}^{\infty} \int_{2^{k}}^{2^{k+1}} H(r) d r \leq 2^{\frac{q+1}{q-1}} \sum_{k=0}^{\infty} b_{k} .
$$

From (9) and (10) we finally obtain

$$
\int_{0}^{\infty} H(r) d r \leq 2^{\frac{q+1}{q-1}}\left(\sum_{k=1}^{\infty} a_{k}+\sum_{k=0}^{\infty} b_{k}\right) \leq 2^{\frac{q+1}{q-1}}(b-a)
$$

since clearly $\left(\sum_{k=1}^{\infty} a_{k}+\sum_{k=0}^{\infty} b_{k}\right) \leq(b-a)$.

\section{Proofs of Theorems 1 And 2}

3.1. Proof of Theorem 1. Assume that there exists a subset $E \subset \partial M$ with compact closure and positive $(n-1)$-Hausdorff measure on which $f$ blows up. Let $r: E \times[0, \delta] \rightarrow \mathbb{R}$ be given by

$$
r(z, s)=\int_{s}^{\delta} \rho(\phi(z, t)) d t
$$


where $\phi$ is the function constructed in Section 2.2 and $\rho$ is as in Section 2.3 Observe that (by Fuglede's theorem) this integral is well defined for $\mathcal{H}^{n-1}$-almost every $z \in E$.

Let us now define $E^{\prime}:=\{z \in E \mid r(z, 0)=\infty\}$ and observe that $\mathcal{H}^{n-1}\left(E \backslash E^{\prime}\right)=$ 0 . This follows from the fact that the inequalities (7) hold for $n$-modulus almost every path in $M$ and from the fact that $f$ blows up on $E$.

Henceforth we assume without lost of generality that $E^{\prime}=E$ and $0<\mathcal{H}^{n-1}(E)<$ $\infty$. Because $f$ is not constant, we may further assume that $\rho(\phi(z, t))>0$ for all $z \in E$ and almost all $0<t<\delta$ (recall that $\rho>0$ almost everywhere if $f$ is not a constant).

Let us define, for every $z \in E$, the function $t_{z}:[0, \infty] \rightarrow[0, \delta]$ to be the inverse of $s \rightarrow r(z, s)$, i.e., $t_{z}=t_{z}(r)$ is defined by

$$
\int_{t_{z}}^{\delta} \rho(\phi(z, t)) d t=r
$$

Next we define $A_{r} \subset M$ to be the set of points $\phi(z, s)$ such that $r(z, s) \leq r$, i.e., $A_{r}:=\phi\left(\left\{(z, s): z \in E^{\prime}\right.\right.$ and $\left.\left.t_{z}(r)<s<\delta\right\}\right) \subset M$.

Then we have from (8) that

$$
\operatorname{Vol}\left(f A_{r}\right) \geq \frac{1}{c \operatorname{deg}(f)} \int_{E} \int_{t_{z}(r)}^{\delta} \rho^{n}(\phi(z, t)) d t d \tau
$$

where $c$ is the constant in inequality (6). Setting $\chi(r):=\left(\frac{r}{\operatorname{Vol}\left(f A_{r}\right)}\right)^{1 /(n-1)}$ and $F_{z}(r):=\int_{t_{z}(r)}^{\delta} \rho^{n}(\phi(z, t)) d t$, we conclude from inequality (11) that

$$
\begin{aligned}
\chi(r) & \leq\left(\frac{c \operatorname{deg}(f) r}{\int_{E} \int_{t_{z}(r)}^{\delta} \rho^{n}(\phi(z, t)) d t d \tau}\right)^{1 /(n-1)}=\left(\frac{c \operatorname{deg}(f) r}{\int_{E} F_{z}(r) d \tau(z)}\right)^{1 /(n-1)} \\
& \leq C_{1} \int_{E}\left(\frac{r}{F_{z}(r)}\right)^{1 /(n-1)} d \tau(z) .
\end{aligned}
$$

Here we have introduced the notation $C_{1}=\left(\frac{c \operatorname{deg}(f)}{\left(\mathcal{H}^{n-1}(E)\right)^{n}}\right)^{1 /(n-1)}$ and we have used Jensen's inequality:

$$
\left(\int_{E} F_{z}(r) d \tau(z)\right)^{-1 /(n-1)} \leq\left(\mathcal{H}^{n-1}(E)\right)^{-n /(n-1)} \int_{E}\left(\frac{1}{F_{z}(r)}\right)^{1 /(n-1)} d \tau(z)
$$

Now integrating $\chi(r)$ and using Fubini's theorem, we obtain:

$$
\begin{aligned}
\int_{0}^{\infty} \chi(r) d r & \leq C_{1} \int_{0}^{\infty} \int_{E}\left(\frac{r}{F_{z}(r)}\right)^{1 /(n-1)} d \tau(z) d r \\
& =C_{1} \int_{E} \int_{0}^{\infty}\left(\frac{r}{F_{z}(r)}\right)^{1 /(n-1)} d r d \tau(z)
\end{aligned}
$$

From Lemma 1 we know that

$$
\int_{0}^{\infty}\left(\frac{r}{F_{z}(r)}\right)^{1 /(n-1)} d r=\int_{0}^{\delta}\left(\frac{\int_{t_{z}(r)}^{\delta} \rho(\phi(z, t)) d t}{\int_{t_{z}(r)}^{\delta} \rho^{n}(\phi(z, t)) d t}\right)^{\frac{1}{n-1}} \rho(\phi(z, t)) d t \leq \delta 2^{\frac{n+1}{n-1}}
$$


hence

$$
\int_{0}^{\infty} \chi(r) d r \leq C_{1} \int_{E} \delta 2^{\frac{n+1}{n-1}} d \tau(z) \leq C_{1} \delta 2^{\frac{n+1}{n-1}} \mathcal{H}^{n-1}(E)<\infty .
$$

We have thus established that if $f$ blows up on $E$, then $\int_{0}^{\infty} \chi(r) d r<\infty$. To conclude the proof of Theorem 1, it remains to relate the function $\chi$ to the isodiametric profile $\eta$ of $N$.

Using (7), one sees that

$$
\operatorname{diam}\left(f A_{r}\right) \leq 2 K^{1 / n} r+\operatorname{diam}(f(E \times\{\delta\})),
$$

i.e., we have an estimate $\operatorname{diam}\left(f A_{r}\right) \leq a r+b$. Hence, by the definition of $\eta$ this implies that $\operatorname{Vol}\left(f A_{r}\right) \leq \eta(a r+b)$ and thus

$$
\chi(r) \geq\left(\frac{r}{\eta(a r+b)}\right)^{1 /(n-1)} .
$$

Since $a r+b \leq 2 a r$ if $r \geq b / a$ and $\eta$ is a monotone function, we have $\eta(a r+b) \leq$ $\eta(2 a r)$ for $r \geq b / a$; it follows that

$$
\chi(r) \geq\left(\frac{r}{\eta(a r+b)}\right)^{1 /(n-1)} \geq\left(\frac{r}{\eta(2 a r)}\right)^{1 /(n-1)}
$$

(for $r \geq b / a$ ) and thus

$$
\int_{b / a}^{\infty}\left(\frac{r}{\eta(2 a r)}\right)^{1 /(n-1)} \leq \int_{b / a}^{\infty} \chi(r) d r<\infty .
$$

This contradicts the hypothesis of Theorem 1 and we conclude that $f$ cannot blow up on $E$.

3.2. Proof of Theorem 2. The proof of Theorem 2 is quite similar to the proof of Theorem 1 but for the sake of completeness, we give a fairly detailed argument. Suppose that $f$ collapses on the relatively compact subset $E \subset \partial M$. We define $r: E \times[0, \delta] \rightarrow \mathbb{R}$ by

$$
r(z, s)=\int_{0}^{s} \rho(\phi(z, t)) d t
$$

where $\phi: E \times[0, \delta] \rightarrow N$ is the map defined in Section 2.2.

Observe that if $E$ has positive $(n-1)$-dimensional measure, then there exists a subset $E^{\prime} \subset E$ with positive $(n-1)$-dimensional measure and a number $\lambda>0$ such that $r(z, \delta)>\lambda$ for all $z \in E^{\prime}$. Indeed, if this were not the case, from Fubini we would get $\int_{\phi(E \times[0, \delta])} \rho d \mu=0$, contradicting the fact that $\rho>0$ a.e.

We may assume without loss of generality that $E=E^{\prime}$, i.e., that $r(z, \delta)>\lambda$ for all $z \in E$. Let us denote by $t_{z}:[0, \lambda] \rightarrow \mathbb{R}$ the inverse of the function $s \rightarrow r(z, s)$, i.e.,

$$
\int_{0}^{t_{z}} \rho(\phi(z, t)) d t=r
$$

We have in particular $t_{z}(r)<\delta$ if $0 \leq r \leq \lambda$. Now let

$$
A_{r}:=\phi\left(\left\{(z, s): z \in E \text { and } 0<s \leq t_{z}(r)\right\}\right) \subset M .
$$


Then, we have as above

$$
\operatorname{Vol}\left(f A_{r}\right) \geq \frac{1}{c \operatorname{deg}(f)} \int_{E} \int_{0}^{t_{z}(r)} \rho^{n}(\phi(z, t)) d t d \tau .
$$

As in the proof of Theorem 1 , we set $\chi(r):=\left(\frac{r}{\operatorname{Vol}\left(A_{r}\right)}\right)^{1 /(n-1)}$ and redefine $F_{z}(r):=$ $\int_{0}^{t_{z}(r)} \rho^{n}(\phi(z, t)) d t$. Then the estimate above together with Jensen's inequality implies

$$
\chi(r) \leq\left(\frac{c \operatorname{deg}(f)}{\left(\mathcal{H}^{n-1}(E)\right)^{n}}\right)^{1 /(n-1)} \int_{E}\left(\frac{r}{F_{z}(r)}\right)^{1 /(n-1)} d \tau(z) .
$$

Integrating $\chi(r)$ and using Fubini's theorem and Lemma 1, we deduce that

$$
\begin{aligned}
\int_{0}^{\lambda} \chi(r) d r & \leq C_{1} \int_{0}^{\lambda} \int_{E}\left(\frac{r}{F_{z}(r)}\right)^{1 /(n-1)} d \tau(z) d r \\
& =C_{1} \int_{E} \int_{0}^{\lambda}\left(\frac{r}{F_{z}(r)}\right)^{1 /(n-1)} d r d \tau(z) \\
& \leq C_{1} \delta 2^{\frac{n+1}{n-1}} \mathcal{H}^{n-1}(E)<\infty
\end{aligned}
$$

where $C_{1}=\left(\frac{c \operatorname{deg}(f) r}{\left(\mathcal{H}^{n-1}(E)\right)^{n}}\right)^{1 /(n-1)}$ as before.

We have thus established that if $f$ collapses on $E$, then $\int_{0}^{\lambda} \chi(r) d r<\infty$. To conclude the proof of Theorem 2 , it remains to relate the function $\chi$ to the isodiametric profile $\eta$ of $N$.

Since $f$ collapses on $E$, we have $\lim _{t \rightarrow 0} \operatorname{diam}(f \circ \phi(E \times(0, t)))=0$, and it follows that

$$
\operatorname{diam}\left(f A_{r}\right) \leq 2 K^{1 / n} r
$$

Let us write $u=2 K^{1 / n} r$, so $\operatorname{diam}\left(f A_{r}\right) \leq u$ and hence $\operatorname{Vol}\left(f A_{r}\right) \leq \eta(u)$. Thus, we get

$$
\chi(r)=\left(\frac{r}{\operatorname{Vol}\left(A_{r}\right)}\right)^{1 /(n-1)} \geq\left(\frac{r}{\eta(u)}\right)^{1 /(n-1)}
$$

from which it follows that

$$
\begin{aligned}
\int_{0}^{\lambda} \chi(r) d r & \geq \int_{0}^{\lambda}\left(\frac{r}{\eta(u)}\right)^{1 /(n-1)} d r \\
& =\left(\frac{1}{2^{n} K}\right)^{1 /(n-1)} \int_{0}^{2 K^{1 / n} \lambda}\left(\frac{u}{\eta(u)}\right)^{1 /(n-1)} d u=\infty
\end{aligned}
$$

This contradiction shows that $f$ cannot collapse on $E$, proving the theorem.

\section{Sharpness of the PREVIOUs RESUlts}

In this section, we show by means of examples that Theorems 1 and 2 are in some sense sharp. Let $M=B^{n}$ be the standard unit ball in Euclidean space with its canonical metric $g_{0}=\sum_{i} d x_{i}^{2}$, and $N=B^{n}$ with a conformal Riemannian metric $h_{\rho}=\rho(x) g_{0}$ where $\rho: B^{n} \rightarrow \mathbb{R}_{+}$is a continuous function. In our examples we will construct suitable continuous functions $\rho$ that can be made smooth. 
The distances and the volumes in the Riemannian manifold $\left(N, h_{\rho}\right)$ are given by

$$
\operatorname{Vol}_{\rho}(E)=\int_{E} \rho^{n} d m_{n} \quad \text { and } \quad d_{\rho}(x, y):=\inf _{\gamma_{x, y}} \int_{\gamma_{x, y}} \rho d s
$$

where $m_{n}$ is Lebesgue $n$-dimensional measure and the infimum is taken over all rectifiable curves in $B^{n}$ joining $x$ to $y$. The identity map $f=I d: M \rightarrow N$ is a conformal diffeomorphism.

We denote by $\eta_{\rho}$ the isodiametric profile of $\left(N, h_{\rho}\right)$ as defined in (1). Finally, we let $B_{\rho}(x, r)=\left\{y \mid d_{\rho}(x, y)<r\right\}$ and $B(x, r)$ be the open balls of radius $r$ centered at $x$ in $N$ and $M$ respectively.

If $\rho$ is spherically symmetric (i.e., there exists a function $\tilde{\rho}:[0,1) \rightarrow \mathbb{R}_{+}$such that $\rho(z)=\tilde{\rho}(|z|)$ for each point $\left.z \in B^{n}\right)$, then $f$ collapses on the entire boundary $\partial M$ if $\lim _{t \rightarrow 1} \tilde{\rho}(t)=0$ and blows up on the entire boundary if $\int_{0}^{1} \tilde{\rho}(t) d t=\infty$. (In the sequel we use the same symbol for the two functions $\rho: B \rightarrow \mathbb{R}_{+}$and $\tilde{\rho}:[0,1) \rightarrow \mathbb{R}_{+}$.)

Theorems 1 and 2 have the following consequences:

Corollary 2. Assume that $f$ collapses on $E \subset \partial M$ where $\mathcal{H}_{n-1}(E)>0$. Then for some $\delta>0$ we have $\int_{0}^{\delta}\left(\frac{r}{\eta_{\rho}(r)}\right)^{\frac{1}{n-1}} d r<\infty$.

Corollary 3. Suppose that $f$ blows up on $E \subset \partial M$, where $\mathcal{H}_{n-1}(E)>0$. Then for some $\alpha>0$ we have $\int_{\alpha}^{\infty}\left(\frac{r}{\eta_{\rho}(r)}\right)^{\frac{1}{n-1}} d r<\infty$.

The next result shows that Corollary 2 is sharp.

Theorem 3. Let $\chi:(0,1) \rightarrow \mathbb{R}_{+}$be any positive decreasing function such that $r \rightarrow \chi(r) r$ is increasing, $\lim _{r \rightarrow 0} \chi(r)=\infty$ and

$$
\int_{0}^{1} \chi(r) d r<\infty
$$

Then there exists a continuous function $\rho$ on $B$ and constants $C, \delta>0$ such that $\chi(r) \leq\left(\frac{C r}{\eta_{\rho}(r)}\right)^{\frac{1}{n-1}}$ for $0<r<\delta$ and $f$ collapses on $\partial B^{n}$.

We will need the following simple result.

Lemma 2. Suppose that $\rho>0$ is continuous and bounded on some subset $A \subset$ $B(0,1)$. If $\frac{\max _{A} \rho}{\min _{A} \rho} \leq \gamma$, then

$$
\operatorname{Vol}_{\rho}(A) \leq\left(\frac{\alpha_{n-1}}{n 2^{n}}\right) \gamma^{n}\left(\operatorname{diam}_{\rho}(A)\right)^{n}
$$

where $\alpha_{n-1}$ is the area of the unit sphere $S^{n-1}$.

Proof. Using the Euclidean isodiametric inequality, we obtain

$$
\begin{aligned}
\operatorname{Vol}_{\rho}(A) & \leq\left(\max _{A} \rho\right)^{n} \operatorname{Vol}(A) \leq \gamma^{n}\left(\min _{A} \rho\right)^{n} \operatorname{Vol}(A) \\
& \leq \gamma^{n} \frac{\alpha_{n-1}}{n 2^{n}}\left(\min _{A} \rho\right)^{n}(\operatorname{diam}(A))^{n} \leq \gamma^{n} \frac{\alpha_{n-1}}{n 2^{n}}\left(\operatorname{diam}_{\rho}(A)\right)^{n} .
\end{aligned}
$$


4.1. Proof of Theorem 3. Let us set $a_{k}:=\chi\left(2^{-k}\right)$ (where $k \in \mathbb{N}$ ). By hypothesis, $\left\{a_{k}\right\}$ is an increasing sequence such that $\left\{2^{-k} a_{k}\right\}$ is decreasing and $\sum_{k \geq 1} 2^{-k} a_{k}<$ $\infty$. Note in particular that $a_{k} \leq a_{k+1} \leq 2 a_{k}$ since $2^{-(k+1)} a_{k+1} \leq 2^{-k} a_{k}$.

Let us choose $k_{0} \in \mathbb{N}$, such that $\sum_{k \geq k_{0}} 2^{-k} a_{k} \leq \frac{1}{2}$ and define a sequence $\left\{t_{k}\right\}_{k \geq k_{0}}$ by $t_{k}:=\left(1-\sum_{j \geq k} 2^{-j} a_{j}\right)$. We then have $\frac{1}{2} \leq t_{k_{0}}<t_{k_{0}+1} \leq \cdots<1$ and $\lim _{k \rightarrow \infty} t_{k}=1$.

We next define $\rho: B(0,1) \rightarrow \mathbb{R}_{+}$to be the (radially) piecewise linear function such that $\rho(z)=\frac{1}{a_{k_{0}}}$ if $|z| \leq t_{k_{0}}$ and

$$
\rho(z):=\frac{2^{k}}{a_{k}}\left(\frac{|z|-t_{k}}{a_{k+1}}-\frac{|z|-t_{k+1}}{a_{k}}\right)
$$

if $t_{k} \leq|z| \leq t_{k+1}$ for some integer $k \geq k_{0}$.

Observe that $\rho$ is continuous and $\lim _{|z| \rightarrow 1} \rho(z)=\lim _{r \rightarrow 0} \frac{1}{\chi(r)}=0$, in particular the mapping $f=I d: B(0,1) \rightarrow B_{\rho}(0,1)$ collapses on the whole boundary $\partial B(0,1)$. The main property of the $\rho$-metric is given in the following

Claim. Let $k \geq k_{0}$. Then $\frac{1}{2} 2^{-k} \leq d_{\rho}(z, \partial B(0,1)) \leq 2 \cdot 2^{-k}$ if and only if $t_{k} \leq|z| \leq$ $t_{k+1}$.

To prove the claim, we observe that $\frac{1}{a_{j}} \geq \rho(z) \geq \frac{1}{a_{j+1}}$ for $t_{j} \leq|z| \leq t_{j+1}$, hence

$$
d_{\rho}(z, \partial B(0,1)) \leq \int_{t_{k}}^{1} \rho(t) d t \leq \sum_{j \geq k} \frac{\left(t_{j+1}-t_{j}\right)}{a_{j}}=\sum_{j \geq k} 2^{-j} \leq 2^{1-k}
$$

and

$$
d_{\rho}(z, \partial B(0,1)) \geq \int_{t_{k+1}}^{1} \rho(t) d t d t \geq \sum_{j \geq k+1} \frac{\left(t_{j+1}-t_{j}\right)}{a_{j+1}}=\sum_{j \geq k+1} \frac{a_{j}}{a_{j+1}} 2^{-j} \geq \frac{1}{2} 2^{-k}
$$

because $\frac{a_{j}}{a_{j+1}} \geq \frac{1}{2}$. This establishes the claim.

We need to show that $\left(\frac{C r}{\eta_{\rho}(r)}\right)^{1 /(n-1)} \geq \chi(r)$ if $0<r<\delta$ for some constants $C, \delta>0$; i.e., that for any subset $A \subset B(0,1)$ such that $\operatorname{diam}_{\rho}(A)=r<\delta$ we have $\operatorname{Vol}_{\rho}(A) \leq \operatorname{Cr} \chi(r)^{1-n}$.

We choose $\delta \in\left(0,2^{-k_{0}}\right)$ so small that $B:=B_{\rho}\left(0, \frac{t_{k_{0}}}{2 a_{k_{0}}}+\delta\right)$ is a compact subset of $B(0,1)$ and we divide the proof into three cases depending on whether $A$ is near the center of $B(0,1)$, near its boundary or in between.

Case 1. Assume that $d_{\rho}(A, 0) \leq t_{k_{0}} / 2 a_{k_{0}}$.

Since $\operatorname{diam}_{\rho}(A)=r \leq \delta$, we have $A \subset B:=B_{\rho}\left(0, \frac{t_{k_{0}}}{2 a_{k_{0}}}+\delta\right) \Subset B(0,1)$; in particular

$$
\frac{\max _{A} \rho}{\min _{A} \rho} \leq \frac{\max _{B} \rho}{\min _{B} \rho}:=\gamma
$$

where $\gamma$ is a constant which does not depend on $A$ or $r$.

By the previous lemma, we then have $\operatorname{Vol}_{\rho}(A) \leq\left(\gamma^{n} \frac{\alpha_{n-1}}{n 2^{n}}\right) r^{n}$. Because $r \chi(r)$ is increasing, we have $r \chi(r) \leq \delta \chi(\delta)$ for $0 \leq r \leq \delta$. This last inequality may be written as $r^{n} \leq(\delta \chi(\delta))^{n-1} r \chi(r)^{1-n}$. Hence

$$
\operatorname{Vol}_{\rho}(A) \leq \operatorname{Cr} \chi(r)^{1-n}
$$


where $C=\left(\gamma^{n} \frac{\alpha_{n-1}}{n 2^{n}}\right)(\delta \chi(\delta))^{n-1}$.

Case 2. Assume that $d_{\rho}(A, 0)>t_{k_{0}} / 2 a_{k_{0}}$ and $d_{\rho}(A, \partial B(0,1)) \geq r$.

Because $\operatorname{diam}_{\rho}(A)=r$ and $d_{\rho}(A, \partial B(0,1)) \geq r$, it follows that there is $s \geq r$ so that $s \leq d_{\rho}(z, \partial B(0,1)) \leq s+r \leq 2 s$ for each $z \in A$.

Define $k$ by $2^{-1-k} \leq s<2^{-k}$; using the Claim above and $2^{-1-k} \leq s \leq$ $d_{\rho}(z, \partial B(0,1)) \leq 2 s<2^{1-k}$, we see that $t_{k} \leq|z| \leq t_{k+1}$.

We thus have $\frac{1}{a_{k+1}} \leq \min _{A} \rho \leq \max _{A} \rho \leq \frac{1}{a_{k}}$; it follows that $\frac{\max _{A} \rho}{\min _{A} \rho} \leq \frac{a_{k+1}}{a_{k}} \leq 2$ and we conclude as in the first case.

Case 3. We finally assume that $d_{\rho}\left(A, \partial B^{n}\right) \leq r$.

Since $\operatorname{diam}_{\rho} A \leq r$ and $d_{\rho}\left(A, \partial B^{n}\right) \leq r$, we have

$$
A \subset\left\{z \in B(0,1) \mid d_{\rho}\left(z, \partial B^{n}\right) \leq 2 r\right\} .
$$

It then follows from the Claim that $A$ is contained in the annulus

$$
A_{k}:=\left\{z \in B(0,1)\left|t_{k} \leq\right| z \mid \leq 1\right\}
$$

where $k \in \mathbb{N}$ is defined by $2^{-k}<2 r \leq 2^{1-k}$. We thus have

$$
\begin{aligned}
\operatorname{Vol}_{\rho}(A) & \leq \operatorname{Vol}_{\rho}\left(A_{k}\right) \leq \alpha_{n-1} \int_{t_{k}}^{1} \rho^{n}(t) d t \\
& \leq \alpha_{n-1} \sum_{j \geq k} \frac{\left(t_{j+1}-t_{j}\right)}{a_{j}^{n}}=\alpha_{n-1} \sum_{j \geq k} a_{j}^{1-n} 2^{-j} .
\end{aligned}
$$

Since $a_{k}^{1-n} \geq a_{j}^{1-n}$ for $j \geq k$, the previous inequality implies

$$
\operatorname{Vol}_{\rho}(A) \leq \alpha_{n-1} a_{k}^{1-n} \sum_{j \geq k} 2^{-j} \leq 4 \alpha_{n-1} a_{k}^{1-n} r
$$

where we have used the estimate $\sum_{j \geq k} 2^{-j}=2^{1-k}<4 r$. Finally, since $2^{-k} \geq r$ and $\chi$ is decreasing, we have $a_{k}=\chi\left(2^{-k}\right) \leq \chi(r)$. Hence

$$
\operatorname{Vol}_{\rho}(A) \leq 4 \alpha_{n-1} \chi(r)^{1-n} r
$$

Corollary 3 is also sharp:

Theorem 4. Let $\chi:(1, \infty) \rightarrow \mathbb{R}_{+}$be any positive function such that $r \rightarrow r \chi(r)$ is decreasing, $\chi(r) / \chi(2 r)$ is uniformly bounded and $\int_{1}^{\infty} \chi(r) d r<\infty$. Then there exists a continuous function $\rho$ on $B^{n}$ and constants $C, L>0$ such that $\chi(r) \leq$ $\left(\frac{C r}{\eta_{\rho}(r)}\right)^{\frac{1}{n-1}}$ for $L<r<\infty$ and $f$ blows up on $\partial B^{n}$.

Proof. Let us set $\beta:=\sup \chi(r) / \chi(2 r)<\infty$. The sequence $c_{k}:=\chi\left(2^{k}\right)$ is decreasing and satisfies $c_{k} / c_{k+1} \leq \beta$ for all $k$. Furthermore $\sum_{k} c_{k} 2^{k}<\infty$.

Choose $k_{0}$ such that $\sum_{k \geq k_{0}}^{\infty} c_{k} 2^{k} \leq \frac{1}{2}$, and define $\left\{s_{k}\right\}_{k \geq k_{0}}$ by $s_{k}:=$ $\left(1-\sum_{j \geq k} 2^{j} c_{j}\right)$. We have $\frac{1}{2} \leq s_{k_{0}}<s_{k_{0}+1} \leq \cdots<1$ and $\lim _{k \rightarrow \infty} s_{k}=1$.

Let $\rho: B(0,1) \rightarrow \mathbb{R}_{+}$be the function such that $\rho(z)=\frac{1}{c_{k_{0}}}$ if $|z| \leq s_{k_{0}}$ and

$$
\rho(z):=\frac{1}{2^{k} c_{k}}\left(\frac{|z|-s_{k}}{c_{k+1}}-\frac{|z|-s_{k+1}}{c_{k}}\right)
$$

if $s_{k} \leq|z| \leq s_{k+1}$ for some $k \geq k_{0}$. 
Observe that $\rho$ is continuous and monotone increasing. Thus

$$
\int_{s_{k_{0}}}^{1} \rho(s) d s \geq \sum_{k=k_{0}}^{\infty} \rho\left(s_{k}\right)\left(s_{k+1}-s_{k}\right)=\sum_{k=k_{0}}^{\infty} \frac{1}{c_{k}}\left(2^{k} c_{k}\right)=\infty .
$$

In particular, the mapping $f=I d: B(0,1) \rightarrow B_{\rho}(0,1)$ blows up the whole boundary $\partial B(0,1)$. We will need the following estimates:

Claim. Let $k \geq k_{0}$. Then $\left(2^{k+1}-2^{k_{0}}\right) \leq d_{\rho}\left(z, B\left(0, s_{k_{0}}\right)\right) \leq \beta\left(2^{k+2}-2^{k_{0}}\right)$ if and only if $s_{k} \leq|z| \leq s_{k+1}$.

We have $\frac{1}{c_{j}} \leq \rho(z) \leq \frac{1}{c_{j+1}}$ for $s_{j} \leq|z| \leq s_{j+1}$, hence

$$
d_{\rho}\left(z, B\left(0, s_{k_{0}}\right)\right) \geq \int_{s_{k_{0}}}^{s_{k}} \rho(s) d s \geq \sum_{j=k_{0}}^{k} \frac{\left(s_{j+1}-s_{j}\right)}{c_{j}}=\sum_{j=k_{0}}^{k} 2^{j}=\left(2^{k+1}-2^{k_{0}}\right)
$$

and

$$
d_{\rho}\left(z, B\left(0, s_{k_{0}}\right)\right) \leq \int_{s_{k_{0}}}^{s_{k+1}} \rho(s) d s \leq \sum_{j=k_{0}}^{k+1} \frac{\left(s_{j+1}-s_{j}\right)}{c_{j+1}}=\sum_{j=k_{0}}^{k+1} 2^{j} \frac{c_{j}}{c_{j+1}} \leq \beta\left(2^{k+2}-2^{k_{0}}\right)
$$

because $\frac{c_{j}}{c_{j+1}} \leq \beta$. This proves the claim.

We need to show that $\left(\frac{C r}{\eta_{\rho}(r)}\right)^{1 /(n-1)} \geq \chi(r)$ if $r \geq L$ for some constants $C, L>$ 0; i.e., that for any subset $A \subset B(0,1)$ such that $\operatorname{diam}_{\rho}(A)=r \geq L$ we have $\operatorname{Vol}_{\rho}(A) \leq C r \chi(r)^{1-n}$. We will choose $L=\max \left\{2^{k_{0}}, 2 \frac{s_{k_{0}}}{c_{k_{0}}}=\operatorname{diam}_{\rho}\left(B\left(0, s_{k_{0}}\right)\right)\right\}$.

We divide the proof into two cases depending on whether $A$ is near the center of $B(0,1)$ or not.

Let us denote by $V_{0}$ the $\rho$-volume of the ball $B_{\rho}\left(0, \frac{s_{k_{0}}}{c_{k_{0}}}\right)=B\left(0, s_{k_{0}}\right)$ (in this ball, the $\rho$-metric is $\frac{1}{c_{k_{0}}}$ times the Euclidean metric, hence $\left.V_{0}=\frac{\alpha_{n-1}}{n 2^{n}} c_{k_{0}}^{-n} s_{k_{0}}^{n}\right)$. Let $A \subset B(0,1)$ be an arbitrary subset of $\rho$-diameter $r \geq L$.

Case 1. Suppose that $d_{\rho}\left(A, B\left(0, s_{k_{0}}\right)\right) \leq r$.

Let us choose $k \geq k_{0}$ such that $\left(2^{k+1}-2^{k_{0}}\right) \leq 2 r \leq \beta\left(2^{k+2}-2^{k_{0}}\right)$. Set $A_{k}:=$ $B\left(0, s_{k+1}\right) \backslash B\left(0, s_{k_{0}}\right)$. We have the volume estimate

$$
\begin{aligned}
\operatorname{Vol}_{\rho}\left(A_{k}\right) & \leq \alpha_{n-1} \int_{s_{k_{0}}}^{s_{k+1}} \rho^{n}(s) d s \leq \alpha_{n-1} \sum_{j=k_{0}}^{k} \frac{\left(s_{j+1}-s_{j}\right)}{c_{j+1}^{n}} \\
& \leq \alpha_{n-1} \beta^{n} \sum_{j=k_{0}}^{k} \frac{\left(s_{j+1}-s_{j}\right)}{c_{j}^{n}} \leq \alpha_{n-1} \beta^{n} c_{k}^{1-n} \sum_{j=k_{0}}^{k} \frac{\left(s_{j+1}-s_{j}\right)}{c_{j}} .
\end{aligned}
$$

Since $\frac{\left(s_{j+1}-s_{j}\right)}{c_{j}}=2^{j}$, we have $\sum_{j=k_{0}}^{k} \frac{\left(s_{j+1}-s_{j}\right)}{c_{j}}=\left(2^{k+1}-2^{k_{0}}\right) \leq 2 r$. We also have $c_{k}=\chi\left(2^{k}\right) \geq \chi(2 r)$ since $\chi$ is decreasing and $2 r \geq\left(2^{k+1}-2^{k_{0}}\right) \geq 2^{k}$. Because $\chi(r) \leq \beta \chi(2 r)$, we have $c_{k} \geq \frac{1}{\beta} \chi(r)$ and the previous inequality then implies

$$
\operatorname{Vol}_{\rho}\left(A_{k}\right) \leq\left(\alpha_{n-1} \beta^{n(n-1)}\right) \chi(r)^{1-n} r .
$$

Using the fact that $d_{\rho}\left(A, B\left(0, s_{k_{0}}\right)\right) \leq r$ and $\operatorname{diam}_{\rho}(A)=r$, we can deduce from the claim that $A \subset A_{k} \cup B\left(0, s_{k_{0}}\right)$, hence

$$
\operatorname{Vol}_{\rho}(A) \leq V_{0}+\left(\alpha_{n-1} \beta^{n(n-1)}\right) \chi(r)^{1-n} r \leq C r \chi(r)^{1-n}
$$


for all $r \geq L$ where $C$ is an appropriate constant.

Case 2. Assume that $d_{\rho}\left(A, B\left(0, s_{k_{0}}\right)\right) \geq r$.

In that case any point $z \in A$ satisfies $t \leq d_{\rho}\left(z, B\left(0, s_{k_{0}}\right)\right) \leq t+r \leq 2 t$ for some $t \geq r$.

Define $k$ by $2^{k} \leq t<2^{k+1}$. Using the Claim above and the estimate $2^{k} \leq$ $t \leq d_{\rho}(z, \partial B(0,1)) \leq 2 t<2^{k+1}$, we see that $s_{k} \leq|z| \leq s_{k+1}$. We thus have $\frac{1}{c_{k}} \leq \min _{A} \rho \leq \max _{A} \rho \leq \frac{1}{c_{k+1}}$; it follows that $\frac{\max _{A} \rho}{\min _{A} \rho} \leq \frac{c_{k}}{c_{k+1}} \leq \beta$.

By Lemma 2, we now have $\operatorname{Vol}_{\rho}(A) \leq\left(\beta^{n} \frac{\alpha_{n-1}}{n 2^{n}}\right) r^{n}$. Because $r \chi(r)$ is decreasing, we have $r \chi(r) \leq L \chi(L)$ for any $r \geq L$. This last inequality implies $r^{n} \leq(L \chi(L))^{n-1} r \chi(r)^{1-n}$ and hence

$$
\operatorname{Vol}_{\rho}(A) \leq \operatorname{Cr} \chi(r)^{1-n}
$$

where $C=\left(\beta^{n} \frac{\alpha_{n-1}}{n 2^{n}}\right)(L \chi(L))^{n-1}$.

\section{Mappings With Densities of POlynomial Behavior}

In this section, we show that Theorems 1 and 2 can be improved if a polynomial behavior of the density $\rho$ is assumed.

Although we could work on manifolds, for the sake of simplicity we continue with the setting of the preceding section, i.e., $f:=I d: M=\left(B^{n}, g_{0}\right) \rightarrow N=\left(B^{n}, h_{\rho}\right)$. We have shown that the integral assumptions (2) and (3) on the isodiametric profile are sharp conditions in Theorems 1 and 2. One can check from the construction that for a "critical" isodiametric function $\eta$ ( $\operatorname{such}$ as $\left.\eta(r)=r^{n}\left(\log \frac{1}{r}\right)^{n-1}\right)$, the function $\rho$ has to decay or grow exponentially fast.

Our next result shows that this exponential behavior is in fact necessary.

Theorem 5. Let $a>0$. There exists an $\epsilon=\epsilon(n, a)>0$ such that:

A) If $\rho$ satisfies

(a) $\rho(\phi(t, z)) \geq C_{0} t^{a}$ for all $z \in \partial M$ and all $0<t<\delta$,

(b) $\eta(r) \leq C_{1} r^{n-\epsilon}$ for all sufficiently small $r$,

then $f$ cannot collapse on a subset $E \subset \partial M$ of positive $(n-1)$-Hausdorff measure.

B) If $\rho$ satisfies

(a) $\rho(\phi(t, z)) \leq C_{0} t^{-a}$ for all $z \in \partial M$ and all $0<t<\delta$,

(b) $\eta(r) \leq C_{1} r^{n+\epsilon}$ for all sufficiently large $r$,

then $f$ cannot blow up on a subset $E \subset \partial M$ of positive $(n-1)$-Hausdorff measure.

Here, $a, C_{0}$ and $C_{1}$ are positive constants and $\eta$ is the isodiametric profile of $N=\left(B^{n}, h_{\rho}\right)$. Recall also that, for $M=\left(B^{n}, g_{0}\right)$, we have $\phi(z, s)=(1-s) z$.

Proof. We only prove part (A); we use the same notations as in Section 3.2. Write again

$$
A_{r}:=\phi\left(\left\{(z, s): z \in E \text { and } 0<s \leq t_{z}(r)\right\}\right) \subset M .
$$

Then we have from (11)

$$
\operatorname{Vol}\left(f\left(A_{r}\right)\right) \geq \frac{1}{c} \int_{E} \int_{0}^{t_{z}(r)} \rho^{n}(\phi(z, t)) d t d \tau
$$


and, by Hölder's inequality,

$$
\int_{0}^{t_{z}(r)} \rho^{n}(\phi(z, t)) d t \geq t_{z}(r)^{1-n}\left(\int_{0}^{t_{z}(r)} \rho(\phi(z, t)) d t\right)^{n} \geq t_{z}(r)^{1-n} r^{n} .
$$

Using the growth condition on $\rho$ we obtain

$$
\frac{a+1}{C_{0}} t_{z}(r)^{a+1} \leq \int_{0}^{t_{z}(r)} \rho(\phi(x, t)) d t=r .
$$

Thus

$$
\int_{0}^{t_{z}(r)} \rho(\phi(z, t))^{n} d t \geq C_{1} r^{n-(n-1) /(a+1)}
$$

where $C_{1}=\left(\frac{C_{0}}{a+1}\right)^{(n-1) /(a+1)}$ and we conclude that

$$
\operatorname{Vol}\left(f\left(A_{r}\right)\right) \geq \frac{C_{1}}{c} r^{n-(n-1) /(a+1)}
$$

The claim follows with any choice of $0<\epsilon<(n-1) /(a+1)$.

The proof of part (B) is obtained similarly; we just need to modify the argument above following the proof in Section 3.1.

The polynomial behavior of $\rho$ assumed in Theorem 5 holds in many situations; in particular in the setting of [2] (cf. Section 1.3 above). The following simple result shows that a Harnack inequality on $\rho$ suffices for this conclusion.

Proposition 1. Suppose that there exists a constant $A$ so that

$$
\frac{1}{A} \leq \frac{\rho\left(x_{1}\right)}{\rho\left(x_{2}\right)} \leq A
$$

whenever $x_{1}, x_{2} \in B\left(x, \frac{1}{2} d\left(x, \partial B^{n}\right)\right)$ for some $x \in B^{n}$. Then, if $\rho$ is continuous, it satisfies the polynomial bounds assumptions of the previous theorem.

The Harnack inequality is equivalent to assuming that $f$ is uniformly quasisymmetric in each ball $B\left(x, \frac{1}{2} d(x, \partial M)\right)$.

Proof. Notice first that each point in $B^{n}$ can be joined to the origin with a chain of no more than $1+\log (1 /(1-|x|))$ balls of the type $B(x,(1-|x|) / 2)$. If $B_{j}$ and $B_{j+1}$ are such balls with non-empty intersection, pick $y \in B_{j} \cap B_{j+1}$. Let $y_{j} \in B_{j}$ and $y_{j+1} \in B_{j+1}$. Then the Harnack inequality applied to the pairs $y_{j}, y$ and $y, y_{j+1}$ shows that

$$
\frac{1}{A^{2}} \leq \frac{\rho\left(a_{j}\right)}{\rho\left(a_{j+1}\right)} \leq A^{2}
$$

Iterating this inequality we conclude that

$$
\rho(x) \leq \rho(0) A^{1+\log (1 /(1-|x|))},
$$

and a polynomial upper bound on $\rho(x)$ follows. A polynomial lower bound follows similarly.

By the above discussion Theorem 5 is a generalization of the result in Section 1.3 . 


\section{AN EXAMPLE IN 3-SPACE}

The purpose of this section is to show that, in dimension 2, the quasi-conformal (identity) mapping associated with the conformal change of metric on the unit disk defined by the function $\rho(x):=(1-|x|)^{\alpha}$ can be essentially realized by a quasiconformal mapping $f_{\alpha}: B^{2} \rightarrow N_{\alpha} \subset \mathbb{R}^{3}$ onto a surface with conical singularities in 3-space.

Consistent with the discussion in Section 5, this mapping is uniformly quasisymmetric in each disk $B(x,(1-|x|) / 2), x \in B^{2}$.

The basic idea of the construction is the following: In the first step one constructs a continuous map $f_{0}: B^{2} \rightarrow N_{0} \subset \mathbb{R}^{3}$ onto a polyhedral surface $N_{0}$ such that $f_{0}$ is bi-Lipschitz when the disk is equipped with the Euclidean distance and the surface $N_{0}$ is equipped with the intrinsic length metric induced by the embedding $N_{0} \subset \mathbb{R}^{3}$.

The map $f_{0}$ satisfies furthermore the following three properties:

1) $\lim _{|x| \rightarrow 1} f_{0}(x)=0 \in \mathbb{R}^{3}$.

2) The images $\gamma_{k}=f_{0}\left(\left\{|x|=1-2^{-k}\right\}\right)$ of the circles of radii $r=1-2^{-k}$ are polygonal Jordan curves contained in the horizontal annulus

$$
2^{-k-1} \leq \sqrt{x_{1}^{2}+x_{2}^{2}} \leq 2^{-k}, \quad x_{3}=2^{-k}
$$

3) The curve $\gamma_{k}$ has length comparable to 1 .

This is possible because the surface is wrinkled, in fact the surface $N_{0}$ looks like an ice cream cone which gets more and more complicated when we approach the tip located at the origin of $\mathbb{R}^{3}$.

An example of such a map is obtained as follows:

We denote by $S(r):=\{|x|=r\} \subset B^{2}$ the circle of radius $r$. Given $j \geq 2$, we denote the regular planar star with $2^{j}$ "petals" reaching from the circle $S\left(2^{-j-1}\right)$ to the circle $S\left(2^{-j}\right)$ by $S_{j}$. We assume that one of the petals has a tip at the point $\left(2^{-j}, 0\right)$. Notice that the boundary of the star is a Jordan curve whose length is comparable to 1 as the length of each petal is comparable to $2^{-j}$. We first define the image of the circle $S\left(1-2^{-j}\right)$ under $f$ to be the image of $S_{j}$ under the map $T_{j}\left(x_{1}, x_{2}\right)=\left(x_{1}, x_{2}, 2^{-j}\right)$. The actual definition of $f$ on the circle $S\left(2^{-j}\right)$ is given as the orientation preserving constant speed parametrization of the star with $f\left(1-2^{-j}, 0\right)=T_{j}\left(2^{-j}, 0\right)$. We then define $f$ in the annular region $A_{j}=$ $B\left(0,1-2^{-j-1}\right) \backslash B\left(0,1-2^{-j}\right)$ by extending $f$ linearly in terms of the radius and angle from the boundary circles. This defines $f$ on $B(0,1) \backslash B(0,3 / 4)$. Define $f$ in $B(0,3 / 4)$ using the given values on $S(3 / 4)$ and so that $f$ is bi-Lipschitz on $\bar{B}(0,3 / 4)$.

It follows directly from the construction that $f$ is a bi-Lipschitz map from the disk onto the manifold when we equip the manifold with the internal manifold metric defined as the infimum of the lengths (in the Euclidean metric) of the curves that join the given points; notice that in polar coordinates the angle on the star is an increasing function of the angle on the circle. Moreover, the image of $B(0,1) \backslash B(0,1 / 2)$ is piecewise flat and the image of each disk $B(x, r)$ with $0<r<(1-|x|) / 2$ has diameter and distance to the origin comparable to $r$ and is contained in the union of a uniformly bounded number of planes.

In the second step, we compose $f_{0}$ with the radial map $\Phi_{\alpha}: \mathbb{R}^{3} \backslash\{0\} \rightarrow \mathbb{R}^{3} \backslash\{0\}$ defined by $\Phi_{\alpha}(x):=x|x|^{\alpha}$ to obtain the actual map $f_{\alpha}:=\Phi_{\alpha} \circ f_{0}: B^{2} \rightarrow N_{\alpha} \subset \mathbb{R}^{3}$. 
Let us denote $A(x, r):=f_{\alpha}(B(x, r)) \subset N_{\alpha}$ where $B(x, r)$ is a disk in $B^{2}$ (with $0<r \leq(1-|x|) / 2)$. Then, for any $\alpha \neq-1$, we have

$$
B\left(f_{\alpha}(x), \frac{1}{C_{1}}(1-|x|)^{\alpha} r\right) \subset A(x, r) \subset B\left(f_{\alpha}(x), C_{1}(1-|x|)^{\alpha} r\right),
$$

and

$$
\frac{1}{C_{2}}(1-|x|)^{2 \alpha} \pi r^{2} \leq \operatorname{area}(A(x, r)) \leq C_{2}(1-|x|)^{2 \alpha} \pi r^{2}
$$

where $C_{1}, C_{2}$ are constants.

Thus $f_{\alpha}: B^{2} \rightarrow N_{\alpha}$ is a quasi-conformal mapping and its Jacobian satisfies a.e.

$$
\frac{1}{C_{2}}(1-|x|)^{2 \alpha} \leq J_{f}(x)=\rho^{2}(x) \leq C_{2}(1-|x|)^{2 \alpha} .
$$

It is now clear, in view of our first example in the introduction that the map $f_{\alpha}$ collapses on the entire boundary $S^{1}=\partial B^{2}$ if $\alpha>0$ and blows up on the entire boundary when $\alpha<-1$.

\section{Remarks.}

1) The surface $N_{\alpha}$ is singular. However, $N_{0}$ is a polyhedral surface and $N_{\alpha}$, being a diffeomorphic image of $N_{0}$, is thus a Riemannian surface with conical singularities. In the neighborhood of a singular point in such a surface, the metric tensor can be written as

$$
d s^{2}=\mathrm{e}^{2 u}|z|^{2 \beta}|d z|^{2}
$$

where $z=x+i y$ is a local coordinate system centered at the singularity, $u=u(z)$ is continuous and $\beta>-1$ (see [14] or [12]). In particular, the surface $N_{\alpha}$ has a singular metric tensor but the conformal structure extends smoothly.

2) In order to obtain volume growth of the type of Theorems 3 and 4 one would need decay (or growth) faster than any polynomial order for $\rho$ in the above construction. We do not know if such a map can be constructed.

3) We also do not know how to construct similar examples in higher dimensions.

\section{ACKNOWLEDGMENT}

Some of the work for this paper was accomplished while the first author was visiting at the University of Jyväskylä. He would like to thank the University and the Mathematics Department for its generous support and warm hospitality. The authors would like to thank the referee for the careful reading of the manuscript.

\section{REFERENCES}

[1] L.V. Ahlfors, On quasi-conformal mappings. J. Analyse Math. 3 (1954), 1-58.

[2] M. Bonk, P. Koskela and S. Rohde, Conformal metrics on the unit ball in Euclidean space. Proc. London Math. Soc. 77 (1998), 635-664. MR 99f:30033

[3] I. Chavel, Riemannian Geometry - A Modern Introduction. Cambridge University Press (1993). MR 95j:53001

[4] L.C. Evans and R.F. Gariepy, Measure Theory and Fine properties of Functions. Studies in Advanced Mathematics (1992). MR 93f:28001

[5] B. Fuglede, Extremal length and functional completion. Acta. Math. 98 (1957), 171-219. MR 20:4187

[6] F.W. Gehring, Rings and quasi-conformal mappings in space. Trans. Amer. Math. Soc. 103 (1962), 353-393. MR 25:3166 
[7] J. Heinonen, T. Kilpeläinen and O. Martio, Nonlinear Potential Theory of Degenerate Elliptic Equations. Oxford Math. Monographs (1993). MR 94e:31003

[8] J. Heinonen and S. Rohde, The Gehring-Hayman inequality for quasihyperbolic geodesics. Math. Proc. Cambridge Philos. Soc. 114 (1993), 393-405. MR 94j:30019

[9] O. Martio and W.P. Ziemer, Lusin's condition ( $N)$ and mappings with nonnegative jacobian. Michigan Math. J. 39 (1992), 495-508. MR 93h:26021

[10] G.D. Mostow, Quasi-conformal mappings in $n$-space and the rigidity of hyperbolic space forms. Publ. Math. IHES 34 (1969), 53-104. MR 38:4679

[11] Yu.G. Reshetnyak, Space Mappings with Bounded Distortion. Translations of Mathematical Monographs 73, 1989. MR 90d:30067

[12] Yu.G. Reshetnyak, Two-dimensional manifolds of bounded curvature. Encyclopedia of Math. Sciences, vol. 70 Geometry IV, Springer 1993. CMP 94:08

[13] S. Rickman, Quasi-regular Mappings. Springer Ergebnisse der Mathematik 26, 1993. MR 95g:30026

[14] M. Troyanov, Prescribing curvature on compact surfaces with conical singularities. Trans. Amer. Math. Soc. 324 (1991), 793-821. MR 91h:53059

[15] M. Troyanov, Parabolicity of Manifolds. Siberian Adv. Math. 9 (1999), 125-150. MR 2001e:31013

[16] J. Väisälä, Lectures on n-Dimensional Quasiconformal Mappings. Springer Lect. Notes. in Math. 229, 1971. MR 56:12260

[17] V.A. Zorich and V.M. Kesel'man, On the Conformal Type of a Riemannian Manifold. Funct. Anal. Appl. 30 (1996), 106-117. MR 97f:31022

Department of Mathematics, St. Olaf College, Northfield, Minnesota 55057

E-mail address: hansonb@stolaf.edu

Department of Mathematics and Statistics, University of Jyväskylä, P.O. Box 35, FIN-40351 JYVÄSKYLÄ, FinLAND

E-mail address: pkoskela@math.jyu.fi

Department of Mathematics, Ecole Polytechnique Federale de Lausanne (EPfl), 1015 Lausanne, Switzerland

E-mail address: marc.troyanov@epfl.ch 